\title{
La semplicità sintattica in prospettiva testuale. Riflessioni a partire dalla Guida alla redazione degli atti amministrativi
}

\author{
Angela Ferrari
}

PUBBLICATO: 19 MARZO 2021

$\mathrm{N}$

el 20 I è uscita la Guida alla redazione degli atti amministrativi. Regole e suggerimenti, frutto della collaborazione tra l'Accademia della Crusca, l'Università di Firenze e l'Istituto di

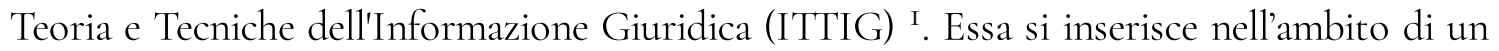
ampio insieme di lavori che, a partire dagli anni Novanta, si pongono l'obiettivo di fornire indicazioni su come 'semplificare' il linguaggio amministrativo in modo da favorirne la comprensione da parte dei suoi destinatari.

Si tratta di una guida complessivamente eccellente, che si articola in più sezioni: un preambolo dedicato ai Principi generali per la redazione dei testi amministrativi; una prima parte intitolata Regole linguistiche per la stesura dei testi amministrativi; una seconda parte che si occupa della Struttura del provvedimento amministrativo; una terza parte che propone Riferimenti ad altri atti, seguita da una serie di Allegati e da un Indice analitico.

Come linguista, mi interessano soprattutto il preambolo e la prima sezione. Sullo sfondo di quanto viene detto nell'introduzione, vorrei in particolare discutere brevemente una delle regole linguistiche proposte, per attirare l'attenzione sul rischio di un fraintendimento importante che puo caratterizzare i consigli forniti in generale da questo tipo di guide. Si tratta del fatto di offrire indicazioni che chiamano in causa la superficie linguistica del testo senza tener conto della loro plausibilità dal punto di vista testuale e comunicativo. È una riflessione doverosa per chi come me si occupa di linguistica testuale e di pragmatica del testo scritto, la quale tuttavia - sia ben chiaro - nulla toglie all'eccellenza complessiva della guida.

\section{Il preambolo comunicativo e le regole linguistiche}

I1 Preambolo della Guida (pp. I5-I7) evoca i cinque principi fondamentali a cui deve sottostare la stesura di un testo amministrativo adeguato dal punto di vista comunicativo:

La redazione dei testi amministrativi risponde a criteri di chiarezza, precisione, coerenza, semplicità, economia. (p. 15)

Nei capoversi successivi, questi criteri sono spiegati in modo articolato:

Un testo è chiaro se i suoi contenuti informativi sono ben riconoscibili e interpretabili da parte del destinatario e se la loro concatenazione logica rispetta uno sviluppo coerente e graduale rispetto alle conoscenze pregresse del destinatario e alle sue aspettative di conoscenza dell'argomento trattato. Chi scrive il testo dovrebbe seguire una progressione informativa rispettosa dei punti cardine di un testo ben formato (Chi?, Che cosa?, Perché?, Dove?, Quando?) in modo da rendere chiaro con la massima evidenza: il soggetto che adotta l'atto, l'oggetto dell'atto e la decisione che è stata presa insieme alle motivazioni che la giustificano, i modi e i tempi di applicazione del testo.

Un testo è preciso se le parole usate e le connessioni logiche tra le frasi risultano univoche ed esplicite. 
La vaghezza e l'ambiguità lessicali, infatti, favoriscono incertezze nell'interpretazione del testo, e connettivi frasali troppo lunghi, arcaici o involuti ne rallentano la comprensione.

Un testo è coerente dal punto di vista linguistico se permette di riconoscere senza equivoci quando ci si riferisce a uno stesso argomento e se permette anche di seguirne lo sviluppo logico.

Un testo è semplice se dà la preferenza a parole conosciute dalla maggior parte dei cittadini o se riesce a parafrasare o a spiegare con sinonimi o esempi gli inevitabili tecnicismi, e se organizza i periodi in modo lineare con poche subordinate e con un uso adeguato della punteggiatura.

Un testo è economico se contiene tutto quello che è necessario e solo quello che è adeguato allo sviluppo del suo contenuto. In particolare, un testo ben costruito è privo di ridondanze, cioè parole e frasi che ripropongono inutilmente concetti già espressi. (pp. 15-16)

Sta naturalmente allo scrivente decidere, sulla base del testo in fieri e del contesto, quali di queste indicazioni devono prevalere in caso di conflitto:

I principi di chiarezza, precisione, coerenza, semplicità ed economia possono, in singoli contesti, entrare in conflitto tra loro. Si tratta di trovare, di volta in volta, il punto di equilibrio tra questi principi. (p. 16)

Come si può notare, sono convocati qui i concetti fondamentali della linguistica del testo: in particolare, l'organizzazione logico-argomentativa del discorso; la sua strutturazione tematicoreferenziale; la sua progressione cognitiva dal dato al nuovo.

Su questo sfondo testuale-comunicativo, si iscrivono poi, nella Parte prima del volume, 29 regole linguistiche, che - si capisce - dovrebbero essere seguite affinché si realizzino le condizioni comunicativo-testuali evocate in precedenza. Queste regole, che si innestano implicitamente sui difetti riconosciuti tradizionalmente al cosiddetto burocratese (si veda, tra gli innumerevoli riferimenti possibili, la sintesi di Lubello 20r6), riguardano i seguenti aspetti della scrittura amministrativa:

Stile 2. Brevità del periodo 3. Il testo e le connessioni tra le frasi 4. Coordinazione congiuntiva e disgiuntiva 5. Tipi di enumerazioni 6. Frasi condizionali e congiunzioni pertinenti 7. Frasi negative 8. Modi, tempi e persone del verbo 9. Uso dei verbi dovere e potere io. Uso della forma attiva, passiva e impersonale dei verbi II. Uso di pronomi e aggettivi I2. Scelta e uso delle parole iz. Termini giuridici o tecnici i4. Interpretazione di termini che hanno diversi significati: contesto e definizioni 15. Arcaismi, latinismi e neologismi i6. Forestierismi i7. Nomi di mestiere, titoli professionali e ruoli istituzionali i8. Coerenza terminologica I9. Uso di abbreviazioni e sigle 20. Uso delle lettere maiuscole 2r. Le maiuscole nei nomi di enti e di luoghi composti da più parole 22. Usi e funzioni della punteggiatura 23. Apostrofo e accento 24. Scrittura dei numeri 25. Date e ore 26. Unità di misura e moneta 27. Simboli convenzionali propri di linguaggi tecnici o scientifici 28. Presentazione grafica del testo 29. Verifica della stesura del testo.

\section{La regola relativa alla Brevitá del periodo}

La seconda regola linguistica, dedicata all'ampiezza e conformazione sintattica del periodo, è così espressa:

Formulare periodi brevi e chiari. 
Usare preferibilmente frasi semplici.

Nei periodi la frase principale precede, preferibilmente, le frasi subordinate che devono essere di numero ridotto. Evitare, se possibile, gli incisi.

Nella stesura di un testo si consiglia di disporre le informazioni in singole frasi e di ordinarle in sequenza logica. È opportuno inoltre iniziare il periodo con la frase principale e far seguire le subordinate. Si raccomanda di adottare in ciascuna frase l'ordine soggetto-verbo-oggetto direttooggetto indiretto (l'utente consegna il modulo $Y$ al responsabile della procedura) e di non inserire frasi o elementi tra il soggetto e il verbo, tra l'ausiliare e il verbo e, in generale, tra un elemento e un altro strettamente collegati (a procedura finita è necessario inviare l'avviso non è necessario a procedura finita inviare l'avviso).

Gli incisi e le frasi tra parentesi costringono il lettore a memorizzare troppi elementi prima di dare un senso compiuto al periodo ed è per questo che si consiglia di limitarne l'uso ai casi strettamente necessari. (p. ı, rilievo originale)

Riguardo alla forma sintattica delle unità linguistiche, si consiglia di scegliere piuttosto frasi semplici (i.e. costituite da una sola proposizione), di costruirle rispettando l'ordine di base del loro nucleo sintattico-semantico (soggetto-verbo-oggetto diretto-oggetto indiretto), e, nel caso in cui si opti per la frase complessa, di collocare la reggente in prima posizione e la subordinata nella posizione successiva.

Ma bisogna fare davvero così? È proprio vero che queste strutture sintattiche sono al servizio di un testo coerente, chiaro e economico? Non sempre. Se si vanno a vedere testi ben scritti - anche quelli che fanno della semplicità sintattica la loro cifra stilistica - ci si accorge che ci sono casi in cui l'oggetto precede il verbo o il soggetto lo segue, e ancora casi in cui la subordinata viene collocata prima della reggente. In tutte queste configurazioni, normalizzare la sintassi porterebbe a costruire testi meno coerenti di quelli originali. Il fatto è che le ragioni del testo non coincidono sempre con le ragioni della sintassi, e la felicità comunicativa degli atti linguistici è legata più alle prime che alle seconde.

\section{Le vere ragioni dell'ordine dei costituenti argomentali nella frase semplice}

Nella frase semplice, l'ordine dei costituenti nucleari - ma anche quello dei costituenti circostanziali è funzionale a tre fattori fondamentali, che possono manifestarsi insieme o singolarmente.

Il primo è la struttura topic-focus dell'enunciato, dove il topic (o tema) è il referente attorno al quale verte la predicazione principale dell'enunciato e il focus è l'informazione più importante dal punto di vista comunicativo. Il referente che funge da topic precede tipicamente la forma verbale, mentre quello che esprime il focus è, sempre tipicamente, in posizione finale. Questa regolarità interpretativa spiega perché a volte è più adeguato che l'oggetto preceda il verbo, o ancora che il soggetto lo segua, come quando alla domanda chi è arrivato? si risponde é arrivato Giorgio. Si noti che questa importante regolarità linguistica vale a meno che non subentrino dispositivi sintattici che la stravolgono, come per esempio la scissione sintattica che colloca il focus in prima posizione (é Giorgio che non sopporto) o la dislocazione a destra, che sposta il topic in posizione finale dislocata (l'ho visto ieri, Giorgio).

Il secondo fattore che guida l'ordine dei costituenti nella frase semplice è di carattere cognitivo: riguarda proprietà come la datità o la novità del referente. Tendenzialmente vengono dapprima le informazioni già date e poi quelle (considerate come) nuove per il lettore. Questo principio va spesso 
di pari passo con quello precedente, nel senso che il topic è spesso dato e il focus è spesso nuovo; ma non sempre: ci sono casi di topic nuovi e casi di foci dati.

Il terzo fattore su cui si fonda la distribuzione dei costituenti frasali è la loro pesantezza fonosintattica. Un costituente molto ricco e complesso dal punto di vista sintattico-lessicale tende a occupare la posizione finale dell'enunciato.

Vediamo due esempi pescando nella saggistica divulgativa, con l'obiettivo di mostrare che l'ordine soggetto-verbo-complemento non è necessariamente quello preferito e da preferire. Il primo esempio è il seguente (rilievo mio):

[incipit assoluto del libro] È cifra caratteristica delle Prime lezioni, e rientra oltretutto nelle mie personali consuetudini di scrittura, la trasparenza del discorso. Di qui la riduzione dei tecnicismi linguistici e la riduzione della bibliografia, collocata in fondo ai capitoli, ai pochi strumenti irrinunciabili (oltre all'esplicitazione dei riferimenti citati a testo). Ma di alcuni tecnicismi non si può fare a meno, tanto più se si sceglie di ricorrere frequentemente al commento di singoli testi [...] (Serianni 2015, p. VII)

Come si può notare, la prima frase sceglie l'ordine verbo-soggetto. Mettere il soggetto in seconda posizione è una scelta adeguata dal punto di vista informativo perché l'obiettivo dell'enunciato è mettere in rilievo la trasparenza del discorso, che è dunque il focus dell'enunciato. È coerente con la strutturazione tematica e logico-argomentativa del testo anche l'anteposizione del complemento di alcuni tecnicismi nell'enunciato successivo in rilievo: se il complemento precede il verbo è perché esso è il topic cognitivamente dato dell'enunciato, come mostra il fatto che viene ripreso a partire dall'enunciato precedente (di qui la riduzione dei tecnicismi linguistici ecc.).

Vediamo ancora il testo seguente:

[...] l'anno successivo sarebbe stata attivata una cattedra anche nell'Università di Roma per Alfredo Schiaffini (I895-1971); [...]

A Schiaffini dobbiamo, tra l'altro, una raccolta di testi fiorentini del Due e Trecento (1926), che intendevano rappresentare una specie di preistoria della prosa d'arte destinata a fiorire col Boccaccio. La raccolta è importante ancora oggi per due motivi: [...] (Serianni 2015, p. 6)

L'enunciato in rilievo ha la struttura oggetto indiretto-verbo - e non verbo-oggetto indiretto -, il che è assolutamente adeguato dal punto di vista comunicativo. Il costituente A Schiaffini è il topic dato dell'enunciato, ed è dunque giusto che compaia in prima posizione: si parla di Schiaffini, e a Schiaffini si è già fatto riferimento nell'enunciato precedente. In questo enunciato il focus coincide con l'oggetto diretto e - anche per la sua pesantezza sintattico-lessicale - è dunque adeguato che chiuda l'enunciato. Non ci stupisce, poi, che questo stesso costituente focale diventi topic dell'enunciato successivo ( $\mathrm{La}$ raccolta), realizzando quella che in linguistica del testo viene chiamata progressione tematica lineare: un focus precedente diventa successivamente topic (Ferrari-Lala-Zampese 202I).

\section{Le vere ragioni dell'ordine delle subordinate nella frase complessa}

L'ordine di principale e subordinate nel periodo è anzitutto legato al tipo di subordinata in gioco. Cosi, le dipendenti relative vengono collocate a ridosso dell'antecedente nominale a cui si agganciano, qualunque sia la sua posizione. Quanto alle subordinate argomentali (soggettive e oggettive), esse 
tendono a seguire la reggente o per ragioni sintattiche (la dislocazione a sinistra di un'oggettiva richiede per esempio la ripresa con un clitico) o perché sono spesso fono-sintatticamente più pesanti e informativamente più ricche della reggente; la loro distribuzione si inverte solo nel caso in cui la subordinata sia cognitivamente data, il che vuole dire, nella sostanza, anaforicamente legata al cotesto precedente. Le dipendenti con funzione circostanziale, quanto a loro, rispondono a principi di tipo comunicativo-testuale. Più precisamente, oltre a quello della complessità sintattico-lessicale, entrano in gioco tre tipi di fattori (che ricalcano in parte quelli validi per l'organizzazione sintattica della frase semplice visti sopra). Il primo riguarda la relazione logica che vige tra le due frasi: cosi per esempio, le subordinate consecutive seguono la reggente perché i loro introduttori le marcano come conseguenze legate a una precedente causa o premessa. Il secondo criterio è di natura informativa: la subordinata è in prima posizione quando funge da quadro semantico della reggente, la quale veicola l'informazione nucleare dell'enunciato. Il terzo criterio è cognitivo: delle due frasi, viene dapprima quella che veicola informazione già data dal cotesto. Come nel caso dei costituenti argomentali della frase semplice, i principi comunicativi che determinano la distribuzione sintattica della frase possono essere bloccati da dispositivi sintattici speciali, come ad esempio la scissione sintattica, che tipicamente colloca la subordinata a inizio enunciato, anche se ha la funzione di nucleo informativo e è saturata da informazioni nuove (è perché è arrabbiato che Giorgio non si è presentato alla riunione).

Vediamo, anche per queste configurazioni, due esempi tratti da saggi divulgativi. Si consideri dapprima il testo seguente:

Quando, verso la fine dell'Impero e soprattutto con le invasioni barbariche, il potere centrale e la stessa unità amministrativa vennero meno e anche l'assetto sociale, economico e culturale delle popolazioni romanizzate mutò profondamente, il processo di trasformazione e di frammentazione linguistica subi unimprovvisa accelerazione. In molte zone dell'Impero il latino fu spazzato via dalle lingue degli invasori [...], in altre [...] diede vita alle nuove lingue romanze, o meglio a una serie di dialetti tra loro più o meno simili, alcuni dei quali avrebbero poi costituito la base delle nuove lingue. (D’Achille 2019, pp. 20-22)

Qui si ha l'ordine subordinata-reggente perché il contenuto semantico della prima funge da quadro rispetto alla seconda, la quale veicola l'informazione più importante dell'enunciato, come mostra il fatto che è proprio il suo contenuto a essere specificato nel cotesto successivo: il secondo enunciato del testo (In molte zone dell'Impero il latino ecc.) illustra infatti quanto è asserito nella reggente (il processo di trasformazione e di frammentazione linguistica subi una brusca accelerazione).

Si veda ancora il seguente estratto:

Se è indubbio che i problemi culturali e le linee di ricerca esistono indipendentemente dal fatto che esista una specifica disciplina di riferimento, è innegabile che l'attivazione di una cattedra universitaria dedicata giovi alla promozione degli studi di quel particolare settore e, prima ancora, ne chiarisca il senso e la direzione. Se è così, l'atto di nascita della storia della lingua risale al r9oo, quando Ferdinand Brunot (1860-1938) fu chiamato alla cattedra di Histoire de la langue française espressamente istituita per lui alla Sorbona. (Serianni 2015, p. 4)

Entrambi gli enunciati sono costruiti attorno allo schema subordinata ipotetica-reggente. Nel primo caso, ciò è dovuto al fatto che siamo di fronte a uno sfruttamento retorico del costrutto condizionale volto a veicolare unopposizione tra due fatti, un effetto retorico che è possibile solo se la subordinata precede la reggente. Nel secondo caso, la dipendente condizionale (Se è cosi) viene prima in quanto il suo contenuto funge da quadro rispetto alla reggente ed è saturata da uninformazione data, come mostra l'espressione anaforica cosi, che riprende quanto è stato detto in precedenza. 


\section{Esempi dalla Costituzione della Repubblica italiana}

Che l'ordine soggetto-verbo-oggetto e quello reggente-subordinata non siano necessari ai fini di una scrittura semplice e trasparente, e che anzi il loro stravolgimento sia garante di coerenza testuale e adeguatezza comunicativa, lo mostra anche la scrittura della Costituzione della Repubblica italiana, considerata in genere come prosa normativa esemplare dal punto di vista della chiarezza (cfr. De Mauro 2006).

Vediamo la prima parte dell'articolo i3:

La libertà personale è inviolabile.

Non è ammessa forma alcuna di detenzione, di ispezione o perquisizione personale, né qualsiasi altra restrizione della libertà personale, se non per atto motivato dell'autorità giudiziaria [...] e nei soli casi e modi previsti dalla legge [...]. (PARTE I, Diritti e doveri dei cittadini, TITOLO I, Rapporti civili, art. I3)

Nel secondo comma, che specifica il primo, abbiamo una struttura verbo-soggetto che si giustifica per via del ruolo comunicativamente focale del soggetto e della sua pesantezza sintattico-lessicale.

Per lo stesso tipo di ragioni viste sopra, troviamo l'ordine verbo-soggetto anche nell'articolo i8:

I cittadini hanno diritto di associarsi liberamente, senza autorizzazione, per fini che non sono vietati ai singoli dalla legge penale [...].

Sono proibite le associazioni segrete e quelle che perseguono, anche indirettamente, scopi politici mediante organizzazioni di carattere militare. (PARTE I, Diritti e doveri dei cittadini, TITOLO I, Rapporti civili, art. I8)

Nell'articolo i7, compare due volte un'anteposizione del complemento in modo da rendere esplicito qual è il topic dell'enunciato (le riunioni) e il suo collegamento con quanto precede:

I cittadini hanno diritto di riunirsi pacificamente e senz'armi.

Per le riunioni, anche in luogo aperto al pubblico, non è richiesto preavviso.

Delle riunioni in luogo pubblico deve essere dato preavviso alle autorità, che possono vietarle soltanto per comprovati motivi di sicurezza o di incolumità pubblica. (PARTE I, Diritti e doveri dei cittadini, TITOLO I, Rapporti civili, art. I7)

Troviamo un posizionamento pre-verbale di un complemento giustificato dal punto di vista informativo-testuale anche nell'articolo 39:

L'organizzazione sindacale è libera $[\ldots]$.

Ai sindacati non può essere imposto altro obbligo se non la loro registrazione presso uffici locali o centrali, secondo le norme di legge. [...] (PARTE I, Diritti e doveri dei cittadini, TITOLO III, Rapporti economici, art. 39)

Per quanto riguarda la distribuzione della subordinata, possiamo andare all'articolo 2r: 
[...] La stampa non può essere soggetta ad autorizzazioni o censure.

Si può procedere a sequestro soltanto per atto motivato dell'autorità giudiziaria nel caso di delitti, per $\mathrm{i}$ quali la legge sulla stampa espressamente lo autorizzi, o nel caso di violazione delle norme che la legge stessa prescriva per l'indicazione dei responsabili.

In tali casi, quando vi sia assoluta urgenza e non sia possibile il tempestivo intervento dell'autorità giudiziaria, il sequestro della stampa periodica può essere eseguito da ufficiali di polizia giudiziaria, che devono immediatamente, e non mai oltre ventiquattro ore, fare denunzia all'autorità giudiziaria. Se questa non lo convalida nelle ventiquattro ore successive, il sequestro s'intende revocato e privo d'ogni effetto. [...] (PARTE I, Diritti e doveri dei cittadini, TITOLO I, Rapporti civili, art. 2I)

In questo caso, se la subordinata temporale (quando vi sia assoluta urgenza ecc.) precede la reggente, è perché fornisce un quadro all'interno del quale vale il contenuto della reggente. E lo stesso vale per la subordinata condizionale seguente (Se questa non lo convalida ecc.); si noti, a questo proposito, che con l'ordine inverso delle frasi la scelta del pronome dimostrativo anaforico (questa) sarebbe stata meno coerente.

\section{Conclusione}

Con la breve riflessione e esemplificazione proposta nelle sezioni precedenti, abbiamo voluto mostrare le difficoltà con cui si scontrano suggerimenti linguistici offerti da guide alla scrittura chiara e semplice che si fermano al piano superficiale della sintassi. Quando non è in gioco la grammaticalità, a definire quali sono le strutture sintattiche da preferire sono criteri di carattere testuale e comunicativo, che tengono conto anche dell'intorno testuale in cui tali strutture si manifestano. La configurazione sintattica della frase, semplice o complessa che sia, codifica valori semantici e informativi: essa è adeguata quando questi valori sono in sintonia con l'obiettivo comunicativotestuale dell'enunciato.

Prendendo lo spunto dalla regola 2 proposta dalla Guida alla redazione degli atti amministrativi uscita nel 2or (e ora in revisione), abbiamo tematizzato il caso dell'ordine dei costituenti argomentali nella frase semplice e quello della distribuzione di reggente e subordinata nella frase complessa. Lo stesso tipo di ragionamento può essere allargato anche a altre strutture sintattiche e testuali. Rimanendo nell'ambito della sintassi, si pensi per esempio alla costruzione passiva, generalmente considerata come una delle cifre del burocratese; essa non va evitata a priori: quando si vuole presentare come topic l'oggetto e focalizzare l'agente, la si può assolutamente scegliere senza che ciò crei problemi interpretativi (in particolare nei testi in cui la dislocazione a sinistra dell'oggetto - che ha gli stessi effetti informativi del passivo - non sia autorizzata per ragioni di registro). Per quanto riguarda la testualità, si può pensare all'espressione del soggetto. Non per forza un testo chiaro deve esplicitare tutti i suoi soggetti sintattici; se la ripresa anaforica è a contatto, la loro implicitezza può essere del tutto adeguata e essere, anzi, al servizio della coesione e dell'unitarietà testuale, come mostra il testo seguente tratto dalla Costituzione (art. 35):

La Repubblica tutela il lavoro in tutte le sue forme ed applicazioni.

Cura la formazione e l'elevazione professionale dei lavoratori.

Promuove e favorisce gli accordi e le organizzazioni internazionali intesi ad affermare e regolare i diritti del lavoro.

Riconosce la libertà di emigrazione, salvo gli obblighi stabiliti dalla legge nell'interesse generale, e tutela 
il lavoro italiano all'estero. (PARTE I, Diritti e doveri dei cittadini, TITOLO III, Rapporti economici, art. 35)

L'esplicitazione di soggetti sintattici testualmente a contatto può creare degli effetti di focalizzazione, che non sono affatto felici dal punto di vista comunicativo.

Peraltro, le conclusioni a cui siamo giunti non valgono solo per la sintassi, ma sono applicabili anche alla punteggiatura. Come abbiamo mostrato in Ferrari et al. 20I8, un'interpunzione che sostiene la chiarezza della scrittura non è solo una punteggiatura che mette in scena la costruzione sintattica dell'enunciato, è anche una punteggiatura che crea architetture informativo-testuali che sono in sintonia con l'obiettivo comunicativo del testo.

Note:

I. Il gruppo di lavoro a cui si deve la sua compilazione è composto da (cito nellordine di apparizione nella Presentazione): Marco Biffi, Angela Frati, Stefania Iannizzotto, Nicoletta Maraschio, Agostino Bultrini, Salvatore Dettori, Barbara Cacelli, Mario Grassia, Graziella Launaro, Giancarlo Minutoli, Sabrina Pellegrino, Paola Ramoino, Paolo Cappelletto, Anna Gentile, Carla Paradiso, Costantino Ciampi, Sebastiano Faro, Raffaele Libertini, Pietro Mercatali, Marina Pietrangelo, Francesco Romano, Pierluigi Spinosa, Dario Zuliani, Andrea Chiari, Gabriella Alfieri, Rosaria Sardo, Massimo Carli, Marta Picchi, Costanza Sanchini, Cecilia Robustelli.

Nota bibliografica:

- Costituzione della Repubblica Italiana (1947), Torino, UTET, 2006.

- D’Achille 2org: Paolo D'Achille, Breve grammatica storica dellitaliano, $3^{\mathrm{a}} \mathrm{ed}$., Roma, Carocci, 2019.

- De Mauro 2006: Tullio De Mauro, Introduzione. Il linguaggio della Costituzione, in: Costituzione della Repubblica Italiana (1947), Torino, UTET, 2006, pp. VII-XXXII.

- Ferrari-Lala-Zampese 202r: Angela Ferrari, Letizia Lala, Luciano Zampese, Le strutture del testo scritto. Teoria e esercizi, Roma, Carocci, 202I.

- Ferrari et al. 2018: Angela Ferrari, Letizia Lala, Fiammetta Longo, Filippo Pecorari, Benedetta Rosi, Roska Stojmenova, La punteggiatura italiana contemporanea. Un'analisi comunicativo-testuale, Roma, Carocci, 2018.

- Guida alla redazione degli atti amministrativi. Regole e suggeerimenti, Firenze, Istituto di teoria e tecniche dell'informazione giuridica del Cnr-Accademia della Crusca, 2orr.

- Lubello 20r6: Sergio Lubello, Usi pubblici e istituzionali dellitaliano, in: Sergio Lubello (a cura di), Manuale di linguistica italiana, Berlin-Boston, De Gruyter, 20I6, pp. 4I7-44I.

- Serianni 20r5: Luca Serianni, Prima lezione di storia della lingua italiana, Roma-Bari, Laterza, 2015.

\section{Cita come:}

Angela Ferrari, La semplicità sintattica in prospettiva testuale. Riflessioni a partire dalla Guida alla redazione degli atti amministrativi , "Italiano digitale", 2021, XVI, 2021/1 (gennaio-marzo)

DOI: $10.35948 / 2532-9006 / 2021.6495$ 